\title{
Transport of colloids along corners: visualization of evaporation induced flows beyond the axisymmetric condition
}

\author{
J. Rodrigo Vélez-Cordero, Bernardo Yáñez Soto, José L. Arauz-Lara \\ Instituto de Física, Universidad Autónoma de San Luis \\ Potosí, Alvaro Obregón 64, 78000 San Luis Potosí, S.L.P., México;
}

August 2, 2016

\section{Supporting Information}

\section{Interfaces obtained with Surface Evolver}

In this study we have shown that it is possible to introduce a geometrical parameter $(a)$ to enforce the substrate angle $\phi$ and contact angle $\theta$ conditions simultaneously. This was possible because the final interfacial shape was assumed to be a section of a solid of revolution. Additionally, this assumption allowed us to expedite the construction of the geometrical model in the finite element program (COMSOL) and solve the Laplacian equation numerically. Rigorously, shapes of liquid-gas interfaces sitting on arbitrary surfaces need to be solved by an energy minimization algorithm, such as that established in the Surface Evolver program [1]. In order to prove that the combination of the angles $\phi$ and $\theta$ at low Bond numbers $(\mathrm{Bo}=0.13)$ indeed yields sections of solids of revolution, we iterated the surface minimization algorithm using this computational package.

We started with a trial shape similar to that used by Berthier \& Brakke in their book, chapter 5 [2], where an initial cube is placed at the corner formed by two orthogonal walls. Relative location, numbering and direction of vertices, edges and facets used in the present problem were similar to those used by these authors. In order to impose the contact angle requirement at two inclined planes, we introduced two energy constraints using the formula $z \pm y \tan \beta=0$ and the force field $\pm \mathcal{T}[z \sin \beta \mp y \cos \beta] i$, where $\mathcal{T}=-\gamma \cos \theta, \beta=\left(180^{\circ}-\phi\right) / 2$ and $\gamma$ is the surface tension. A content vector field of the form $-x z j$ was also necessary to recover the contribution of neglected facets and compute the droplet's volume.

The final shape for some $\theta, \beta$ combinations are shown in the figure below. Note that the pair $\theta=55^{\circ}, \beta=35^{\circ}\left(\phi=110^{\circ}\right)$, used in the manuscript to obtain the geometry depicted in Figure 2b, indeed recover the shape of a section of an ellipsoid of revolution. In general, 
for all the combinations in which $\theta>\beta$, the shape of the final interfaces also resembles sections of spheroids. For $\theta \approx \beta$, however, the interface become flat or experience a change in the curvature's sign; in such conditions the approximations considered in the paper are obviously no longer valid.

\section{References}

[1] The Surface Evolver by Kenneth A. Brakke, Mathematics Department, Susquehanna University,

http://facstaff.susqu.edu/brakke/evolver/html/evolver.htm\#doc-top

[2] J. Berthier and K.A. Brakke, The Physics of Microdroplets, 2012, Wiley, Chapter 5, also available at:

http://facstaff.susqu.edu/brakke/aux/physicsofmicrodroplets/chap5/chap5.html

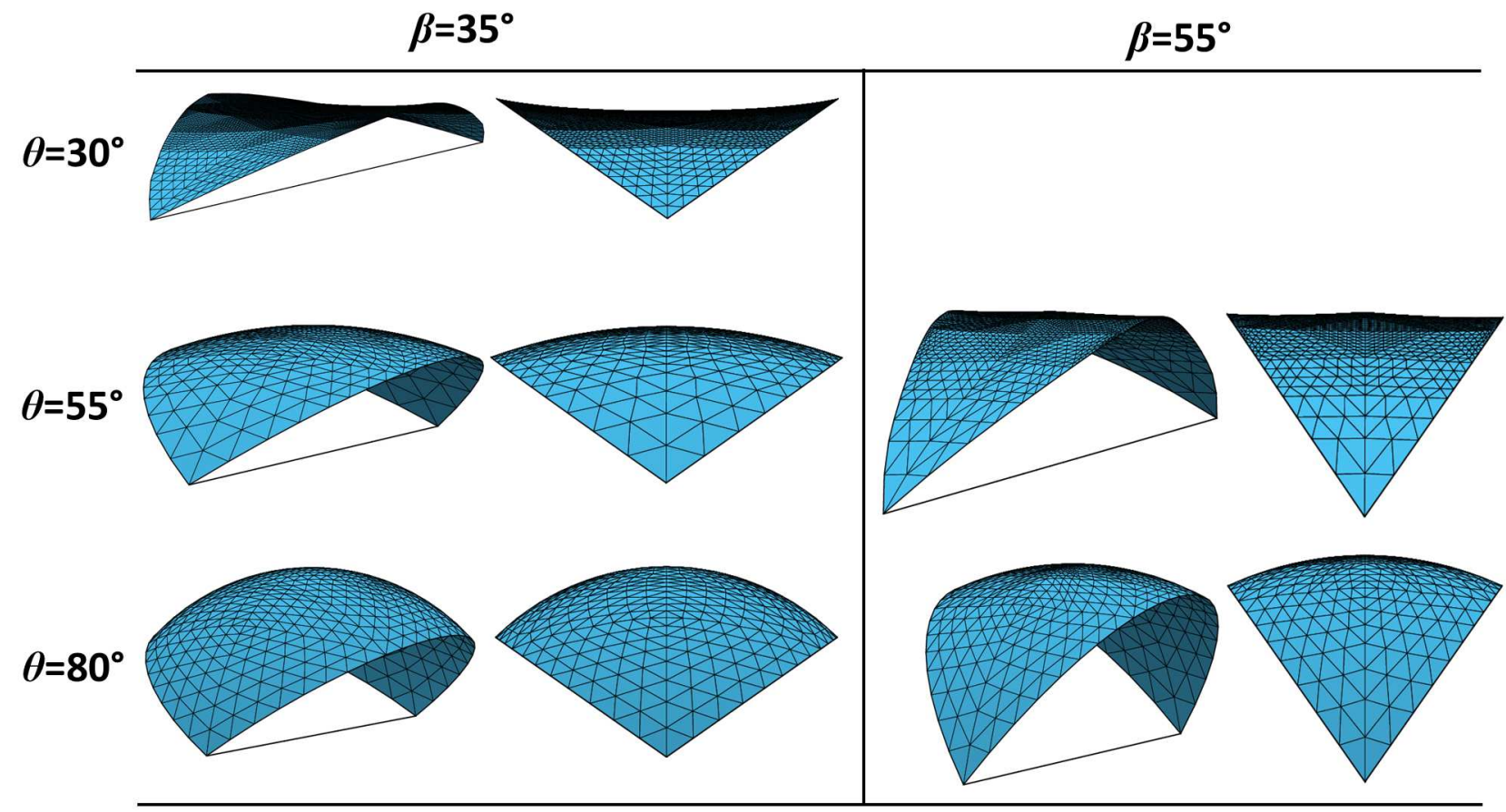

Figure 1: Interfacial shapes obtained with Surface Evolver for different combinations of the contact angle $\theta$ and substrate angle $\beta$. The surface tension value was that of water, $72 \mathrm{mN} / \mathrm{m}$, and the length scale was $1 \mathrm{~mm}$, i.e., less than the capillary length of water, $(\gamma / \rho g)^{1 / 2}=$ $2.7 \mathrm{~mm}$. The inclined solid walls are not shown, only the gas-liquid interfaces which are depicted in blue. Gravity effects were neglected due to low Bond numbers. 